\title{
Evaluation of Supercapacitors Effects on Hybrid Energy Systems for Automotive
}

\author{
Carmen Lungoci and Elena Helerea \\ Department of Electrical Engineering, Transilvania University of Brasov, \\ 1-3 Politehnicii Street, Brasov, Romania \\ lungoci@unitbv.ro
}

\begin{abstract}
This work aims at evaluating the effects of the supercapacitors presence in hybrid energy systems used in automotive. The design and the electrical schema of a hybrid energy system that contains batteries and supercapacitors and propel a synchronous motor are purposed. The motor operating regime is described, detailing the drive evolution of the cycle speed imposed. In these conditions, to model the systems behavior, simulations developed in Matlab/Simulink environment are carried out. Two energies management strategies for the ensemble energy system-motor are implemented. Simulations are done and the energy management is discussed, making the comparative analyses. Applying a current control strategy on the supercapacitors, under two working conditions, functional diagrams are showed and compared. The results obtained highlight the advantages of the supercapacitors.
\end{abstract}

Keywords: Supercapacitors, energy systems, energy management, automotive, simulations.

\section{Introduction}

Nowadays, in transport the use of hybrid energy systems represents an important current topic [1] [2]. In a hybrid vehicle, the most utilized batteries are the Ni-Mh and Li-Ion, presenting advantages due to their technology. But energy recovery using a traditional system composed by batteries (SB) is a constraint. The weight, volume and the price of the batteries reduce the efficiency of the system. From this reason, there are many researches to optimize the architecture and the structure of an energy system [3][4].

The useful strategy adopted is an energy system containing many energy sources. Among the existing solutions, the hybrid energy system using batteries and supercapacitors (SBSC) is proposed and analyzed. This work is aims to develop a SBSC system for electrical traction. The architecture of this system is proposed and the studies regarding the structure, functionality and modeling on it are presented. Using the models for the elements components of the system, developed by the authors in the previous papers, the modeling and simulations are realized, in order to obtain the electrical parameters: current, power, energy, in the test conditions of functioning, imposed by a proposed drive cycle. The necessary power resulted is ensured by 
the hybrid energy system, applying two energy management strategies. In this way, the effects brings by the supercapacitors presence in the energy system are finally underlined.

\section{Contribution to Technological Innovation}

The automotive field requires new technological innovations regarding energy demands inside of subsystems. This trend is putting increasing pressure on energy systems from a vehicle to be efficient and run for long periods. From technical point of $\mathrm{vu}$, they must be able to store and deliver enough energy and power.

Batteries are preferred for most applications because of their superior capability to store energy. The disadvantage consist is the poor ability to deliver power.

Supercapacitors offer a combination of high power and good energy performance parameters with commercial relevance. They are very high surface area activated carbon capacitors that use a molecule-thin layer of electrolyte [5]. This new technology gives them the property to storage energy proportional to the charge surface area. So, they are able to hold a very high charge which can be released in a controlled manner.

Taking in account the batteries and the supercapacitors properties, there is a strong trend in automotive towards hybridizing both energy sources, making the energy system more highly and efficient functional from the point of vu of the energy management. The last technological advances in the applications to automobiles are based on this ability of supercapacitors to complete with the batteries.

\section{State of Art}

In hybrid vehicles, the load profile is characterized by a low value of a main power with big picks of power requested in transient regimes. In these conditions, a hybrid energy system that contains supercapacitors near batteries (SBSC) ensures performing answers of power, higher like in SB systems.

There are researchers which evaluated the effects of the supercapacitors in the hybrid energy systems and they developed and realized practically the SBSC systems [4] [5].

The studies from the literature proved the advantages of SBSC systems use, in which batteries ensure a constant voltage and the role of supercapacitors is to deliver/storage the transient picks of power.

In order to optimize the structure, to improve the functionality and to grow the energy efficiency of the SBSC systems used in automotive, new analyses are necessary, the improvement of the existing models is required and the development of new models is expected.

\section{Research Contribution and Innovation}

In the present research improvements on the energy systems used in automotive are proposed. The main objectives of this work are: 
- To present the ensemble hybrid (batteries-supercapacitors)-synchronous motor;

- To simulate the functioning regimes under different running conditions;

- To implement two energies management in order to highlight the contribution of the supercapacitors in automotive presented system.

\subsection{System Description}

The architecture of the proposed supply/propulsion system consists of a batteries pack and a supercapacitors pack, being utilized in a hybrid vehicle (Fig. 1). The battery pack is directly connected to the DC bus, providing a constant voltage for the system. Mechanical load of the system is represented by the inertia and brakes of the vehicle. Propulsion is ensured by MSA traction motor. The DC/AC inverter, coupled to MSA, transforms the electrical DC power provided on the bus into AC power and controls its parameters.

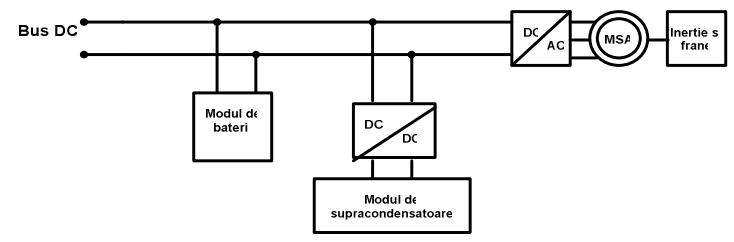

Fig. 1. Architecture of the power system used in hybrid electrical vehicle

The power supplying the inverter is delivered by the both: main and the secondary power source. Supercapacitors pack represents the secondary power source, ensuring optimal performance for the motor in punctual operation regimes. The bidirectional DC/DC converter connects the supercapacitors pack to DC bus, controlling current flow and allowing energy recovery while breaking. The functioning regimes of the motor are modeled according to the proposed running drive cycle - 3ECE. The entire power demand by the motor is ensured by the batteries and supercapacitors pack, designed according to the speed cycle.

The aim of simulations is to obtain a quick and consistent response to transient power requests from the motor, in order to obtain a high energy efficiency of the entire system. The model used for simulations includes a batteries pack consisting of 23 elements, providing a constant voltage in amount of $280 \mathrm{~V}$. Supercapacitors pack introduced in the simulation model has the following parameters: total capacity in amount of $5.8 \mathrm{~F}$, voltage across terminals $150 \mathrm{~V}$ and internal resistance $0.19 \Omega$. Electrical propulsion motor is a permanent magnet synchronous motor, with the following parameters: $U_{\mathrm{n}}=230 \mathrm{~V} ; I_{\mathrm{n}}=42,9 \mathrm{~A} ; M_{\mathrm{n}}=35 \mathrm{Nm} ; n_{\mathrm{n}}=3300 \mathrm{rpm} ; P_{\mathrm{n}}=12,1 \mathrm{~kW}$. Energy efficiency is to be determined in simulations, by analyzing obtained responses.

Two energy management strategies are presented:

1. The necessary of the electrical power requested by the motor is entirely ensured by the batteries (SB system), during the drive cycle period;

2. The same necessary of power is ensured by the batteries and the supercapacitors. 
The energy management is treated, observing the temporal responses obtained by simulations under the under drive cycle conditions for each case above. Running cycles set (3ECE) is composed from several European urban running cycles, containing different acceleration, constant speed and breaking phases. There are presented three levels of rotational speed for the motor: $1100 \mathrm{rot} / \mathrm{min}, 2200 \mathrm{rot} / \mathrm{min}, 3300$ rot/min, the motor dynamics having easy accelerations and breakings.

\subsection{Energy Management Using SB}

The following work assumptions have been considered:

- The unique energy source used is the pack of batteries;

- The entire electrical power requested by the motor is ensured by the batteries and the braking energy of the motor is completely taken by the batteries;

- The model of batteries is an internal resistance in series with an electromotive voltage source;

- For the synchronous motor was used the experimental model developed by the authors and detailed in [6].

In Fig. 2 the necessary of current $\left(I_{\text {bus }}\right)$ variation during the 3ECE cycle is presented. It can be observed the many peaks of current corresponding when crossing to another level of speed. In Fig. 3 the current variation curve on the batteries during the same cycle are obtained. Fig. 4 shows the electrical power required by the traction motor during the cycle and Fig. 5 presents the power electrical variation at the pack of batteries in the same period. Graphics shows that during the running cycle, batteries must ensure all the power picks required by the motor, which implies their overload.

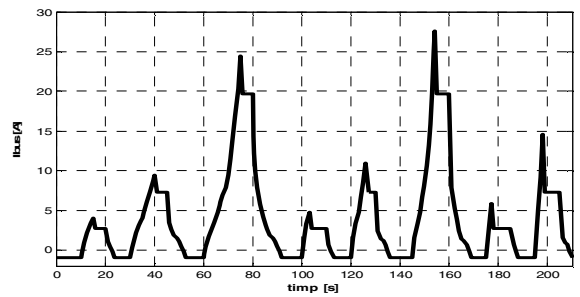

Fig. 2. Motor current $I_{\text {bus }}$ during $3 E C E$ cycle

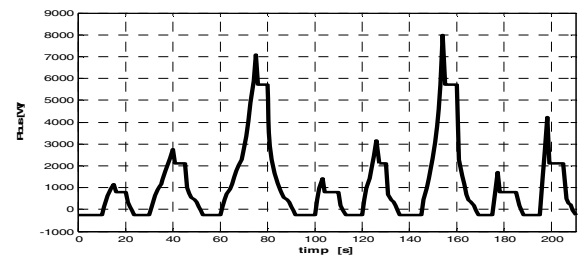

Fig. 4. Electrical motor power $P_{\text {bus }}$

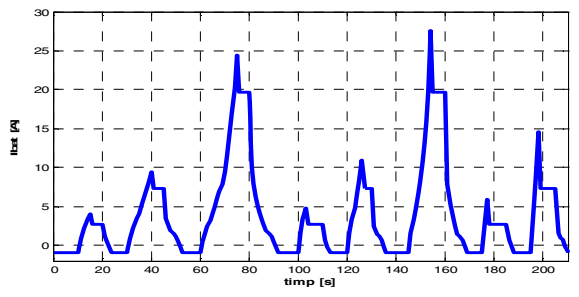

Fig. 3. Current $I_{\text {bat }}$ of batteries during cycle

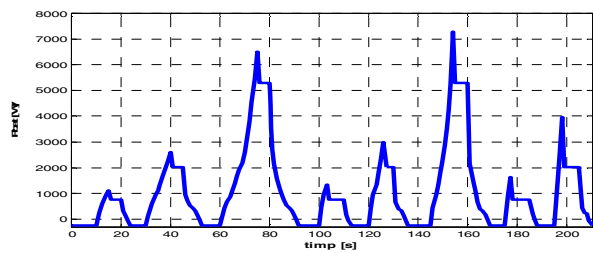

Fig. 5. Electrical batteries power $P_{\text {bat }}$ 
On the other hand, the batteries must take the entire breaking power reinserted by the motor in the DC bus, which is practically impossible. The SB system is not sufficient to ensure the motor functionality, being required to use a hybrid energy system, like SBSC, that can answer efficiently of the dynamics of the motor.

\subsection{Energy Management Using SBSC}

The electrical model of the supercapacitors used in simulations is composed by a variable capacity in series with a resistance. The pack of batteries is simple modeled by an ideal voltage source. The synchronous motor has the experimental model detailed by the authors in the previous works [6]. The functioning regimes of the motor are given by the running drive cycle 3ECE. The power control principle utilized for this SBSC, which establishes the contribution of each source in supplying the motor, is based on the separation of medium power demand from the transitory power demand and consists of a first order low pass filtering function. The filter is applied to the current supplying the motor obtaining a reference output current for controlling the supercapacitors pack. In order to implement control strategy for the supercapacitors pack, the transfer function of a first order filter has been utilized. To highlight the filter action on the system functionality, the time constant will take two values: first, $3 \mathrm{~s}$, than simulations are repeated for value of $6 \mathrm{~s}$. Simulations has been done using the Matlab Simulink software.

The motor current is in Fig. 6. In Fig. 7 is layout the current variation that must be ensured by the pack of batteries, when using the constant time filter $\tau=3 \mathrm{~s}$. In Fig. 8 is shown the variation of the current when $\tau=6 \mathrm{~s}$. Fig. 10 shows the reference current applied to the pack of supercapacitors for $\tau=3 \mathrm{~s}$, and in Fig. 11 for time constant $\tau=6 \mathrm{~s}$. The reference current is resulted after the filter at the converter entry, when batteries and supercapacitors are used together.

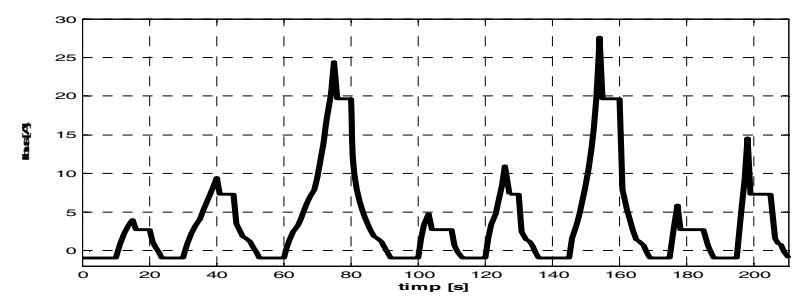

Fig. 6. Current $I_{\text {bus }}$ at the traction motor

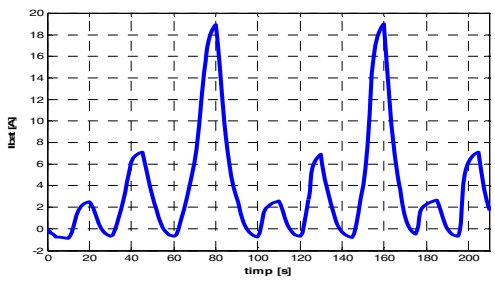

Fig. 7. Batteries current $I_{\text {bat }}$ for $\tau=3 \mathrm{~s}$

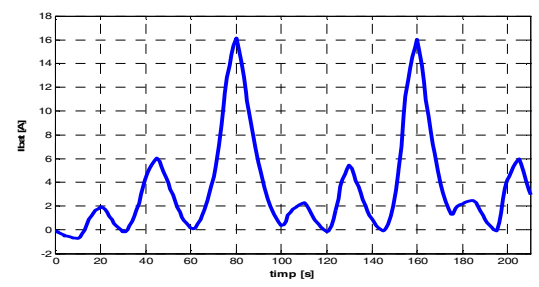

Fig. 8. Batteries current $I_{\text {bat }}$ for $\tau=6 \mathrm{~s}$ 


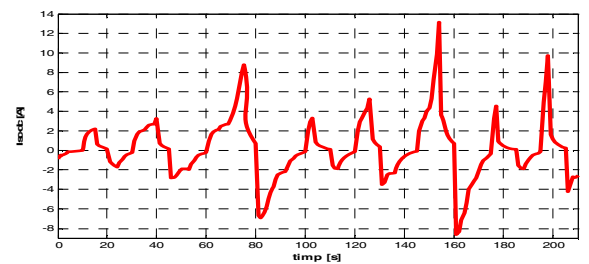

Fig. 9. Reference converter current, $\tau=3 \mathrm{~s}$

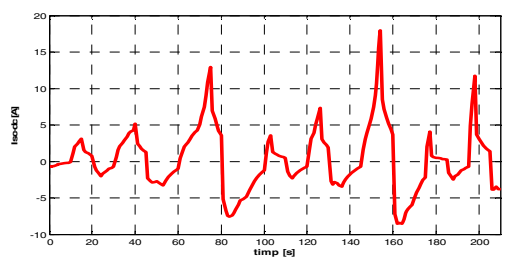

Fig. 10. Reference converter current, $\tau=6 \mathrm{~s}$

The graphics prove an important filter of the current delivered by the batteries. Supercapacitors take efficiently the power picks and batteries are smoothed. Graphics prove a better management of current sharing on both energy sources, which ensures together the necessary of electrical energy of the motor.

\section{Discussions of Results}

In this section, a comparative analyze of graphics in both energy management strategies is done. The power variation curves for the ensemble SBSC-synchronous motor are detailed. In Fig. 11 is represented the requested power by the motor.

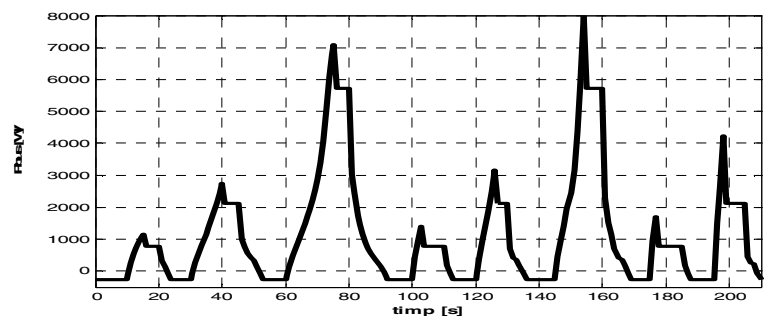

Fig. 11. Power $P_{\text {bus }}$ at the motor terminals

In Fig. 12, Fig. 13 and Fig. 14 are analyzed the results obtained after the utilization of both energy management strategies (SB and SBSC) and after utilization of the power control strategy on the supercapacitors. Therefore, is presented the power variation on the batteries terminals in the three cases:

1. using SB like energy management strategy;

2. using SBSC like energy management strategy and a power management strategy with a filter having $\tau=3 \mathrm{~s}$;

3. using SBSC like energy management strategy and a power management strategy with a filter having $\tau=6 \mathrm{~s}$. 


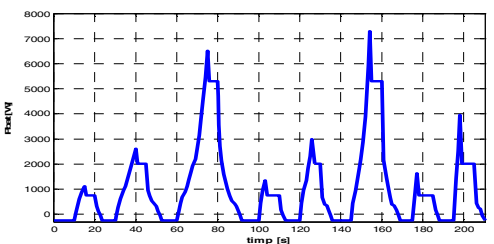

Fig. 12. Batteries power $P_{\text {bat }}$, SB-motor

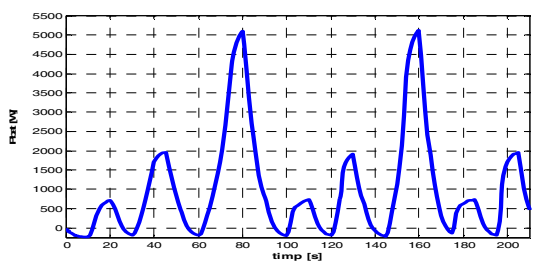

Fig. 14. Batteries power $P_{\text {bat }}$,SBSC-motor, with filter constant $\tau=3 \mathrm{~s}$

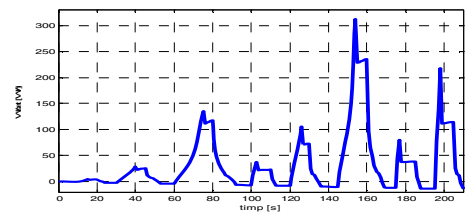

Fig. 13. Batteries energy $W_{\text {bat }}$, SB-motor

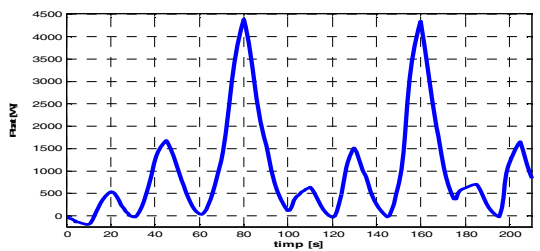

Fig. 15 Batteries power $P_{\text {bat }}$, SBSC-motor, with filter constant $\tau=6 \mathrm{~s}$

The energy management is underlined from the energy variation curves resulted from simulations in the Fig. 13, Fig. 16 and Fig. 17.

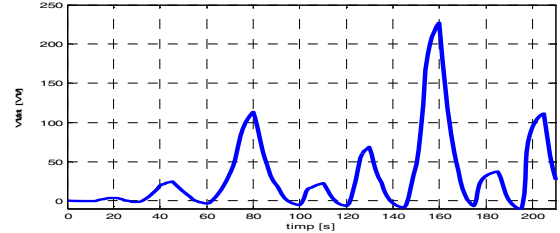

Fig. 16. Batteries energy $W_{\text {bat, }}$ SBSC motor, $\tau=3 \mathrm{~s}$

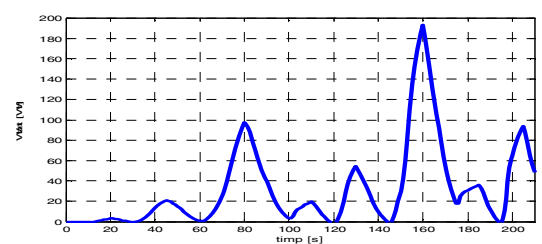

Fig. 17. Batteries energy $W_{\text {bat }}$, SBSC motor, $\tau=6 \mathrm{~s}$

Comparing the graphics obtained, it can be deduced:

1. When using SB systems, power and energy on batteries have high values and high picks, that crave for batteries;

2. Using SBSC systems, the transient picks of power and energy on batteries are smoothed;

3. Growing-up the time constant value of the filter used for power control strategy on supercapacitors, the power/energy on batteries becomes smaller and smoother.

Therefore the contribution of the supercapacitors in the energy deliver/storage processes became more important and the system energy efficiency is improved.

Simulations effected highlight the effects of supercapacitors in the hybrid energy system used in automotive. With this research there are certified: 
- the validity of the SB and SBSC system models;

- the validity of the energy management strategy with SBSC;

- the validity of the control strategy of the supercapacitors pack based on power sources separation;

- the contribution bring by the supercapacitors in hybrid energy systems.

\section{Conclusions and Further Works}

The use of SBSC as power control strategy leads to an optimized model of the propulsion system, in accordance to requirements. The presence of supercapacitors in power supply/recovery systems used for electric traction increases battery lifetime and allows a fair design in terms of weight and volume. Therefore, the entire system can be design at lower power requirements, power dimensioning being separate from energy dimensioning of components, ensuring therefore increase of efficiency, reduction of dimensions, costs and weight. The advantages offered by power systems with batteries and supercapacitors recommend them for applications in transportation, especially where high power peaks are required.

The paper presented an analysis regarding power control and energy management when utilizing SB and than a SBSC-synchronous motor system, in functioning conditions imposed by standard 3ECE running cycles.

A command strategy has been implemented for the supercapacitors pack in order to optimize system performance and energy efficiency.

Results of the analysis prove that supercapacitors particularly reduce current peaks applied to/from the battery and improve acceleration characteristics, range and efficiency of the vehicle, especially when the running cycle determines a lot of current peaks.

For the future, experimental results are expected and new models for the components of system will be implemented.

\section{References}

1. Chan, C., Chan, K.: Modern Electric Vehicle Technology. Oxford University Press, Oxford (2006)

2. Yap, H., Schofield, C., Bingham, C.: Hybrid energy/power sources for electric vehicle traction systems: The Institution of Electrical of Electrical Engineers. Michael Faradya House, Stevenage, SGI 2AY (2004)

3. Steiner, M., Scholten, J.: Energy storage on board of railway vehicles. In: ESSCAP, Belfort, France (2004)

4. Livint, G., Gaiginschi, R., Horga, V., Drosescu, R., Chiriac, G.: Vehicuel electrice hibride. Venus, Iasi (2006)

5. Lungoci, C., Mailat, A., Helerea, E.: Eco-design of a hybrid energy system for electric vehicles. In: 2nd International Conference on Sustainable energy, Brasov, pp. 438-446 (2008)

6. Lungoci, C., Bouquain, D., Miraoui, A., Helerea, E.: Modular test bench for a hybrid electric vehicle with multiples energy sources. In: 11th IEEE International Conference on Optimization of Electrical and Electronic Equipment, Brasov, pp. 299-306 (2008) 